\title{
Geodesic Object Representation and Recognition
}

\author{
A. Ben Hamza and Hamid Krim \\ Department of Electrical and Computer Engineering \\ North Carolina State University, Raleigh NC 27695, USA
}

\begin{abstract}
This paper describes a shape signature that captures the intrinsic geometric structure of $3 \mathrm{D}$ objects. The primary motivation of the proposed approach is to encode a 3D shape into a one-dimensional geodesic distribution function. This compact and computationally simple representation is based on a global geodesic distance defined on the object surface, and takes the form of a kernel density estimate. To gain further insight into the geodesic shape distribution and its practicality in $3 \mathrm{D}$ computer imagery, some numerical experiments are provided to demonstrate the potential and the much improved performance of the proposed methodology in $3 \mathrm{D}$ object matching. This is carried out using an information-theoretic measure of dissimilarity between probabilistic shape distributions.
\end{abstract}

Keywords: Geodesic shape distribution, 3D object representation and matching, Jensen-Shannon divergence.

\section{Introduction}

Three-dimensional objects consist of geometric and topological information, and their compact representation is an important step towards a variety of imaging applications including indexing, retrieval, and matching in a database of 3D models. The latter will be the focus of the present paper. There are two major steps in object matching. The first step involves finding a reliable and efficient shape representation or descriptor, and the second step is the design of an appropriate dissimilarity measure for object comparison between shape representations.

Most three-dimensional shape matching techniques proposed in the literature of computer graphics, computer vision and computer-aided design are based on geometric representations which represent the features of an object in such a way that the shape dissimilarity problem reduces to the problem of comparing two such object representations. Feature-based methods require that features be extracted and described before two objects can be compared. Among featurebased methods, one popular approach is graph matching, where two objects are represented by their graphs composed of vertices and edges. An efficient representation that captures the topological properties of $3 \mathrm{D}$ objects is the Reeb graph descriptor proposed by Shinagawa et al. [1. The vertices of the Reeb graph are the singular points of a function defined on the underlying object 12 . These singularities carry important information for further operations, such as image registration, shape analysis, surface evolution and object recognition $[3,4,5]$. 
An alternative to feature-based representations is the shape distribution developed by Osada et al [6]. The idea here is to represent an object by a global histogram based on the Euclidean distance defined on the surface of the object. The shape matching problem is then performed by computing a dissimilarity measure between the shape distributions of two arbitrary objects. This approach is computationally stable and relatively insensitive to noise. Because of unsuitability of the Euclidean distance when dealing with nonlinear manifolds, the shape distribution, however, does not capture the nonlinear geometric structure of the data.

In this paper, we propose a new approach for object matching based on a global geodesic measure. The key idea behind our technique is to represent an object by a probabilistic shape descriptor called geodesic shape distribution that measures the global geodesic distance between two arbitrary points on the surface of an object. In contrast to the Euclidean distance which is more suitable for linear spaces, the geodesic distance has the advantage to be able to capture the (nonlinear) intrinsic geometric structure of the data. The geodesic shape distribution may be used to facilitate representation, indexing, retrieval, and object matching in a database of 3D models. More importantly, the geodesic shape distribution provides a new way of looking at the object matching problem by exploring the intrinsic geometry of the shape. The matching task therefore becomes a one-dimensional comparison problem between probability distributions which is much easier than comparing 3D structures. Object matching may be carried out by dissimilarity measure calculations between the corresponding geodesic shape distributions, and it is accomplished in a highly efficient way.

Information-theoretic measures provide quantitative entropic divergences between two probability distributions. A common entropic dissimilarity measure is Kullback-Leibler divergence [7] which has been successfully used in many applications including indexing and image retrieval [8]. Another entropy-based measure is the Jensen-Shannon divergence which may be defined between any number of probability distributions [9], and it has been applied to a variety of signal/image processing and computer vision applications including DEM image matching [10], and ISAR image registration [11].

The rest of this paper is organized as follows. The next section is devoted to the problem formulation. Section 3 describes the representation step of our proposed technique. In Section 4, we present the Jensen-Shannon divergence and show its attractive properties as a dissimilarity measure between probability distributions. In Section 5, we provide numerical simulations to show the power of the geodesic shape distribution for $3 \mathrm{D}$ object matching. And finally, Section 6 contains some conclusions and describes a brief outline of possible future work.

\section{Problem Statement}

Three-dimensional objects are usually represented as triangular meshes in computer graphics and geometric-aided design. A triangle mesh is a pair $\mathbb{M}=(\mathcal{V}, \mathcal{T})$, where $\mathcal{V}=\left(\boldsymbol{v}_{1}, \ldots, \boldsymbol{v}_{m}\right)$ is the set of vertices, and $\mathcal{T}=\left(T_{1}, \ldots, T_{n}\right)$ is the set of triangles. 
In scientific visualization and analysis, a triangle mesh is too large to be examined without simplification. One way to overcome this limitation is to represent a triangle mesh by surface features which can easily be computed and which effectively characterize the global surface shape. The centroids of the set of triangles $\mathcal{T}$ are desirable features that can efficiently be computed and which have a global significance for the surface shape representation as illustrated in Fig. [1] In addition, there is a well defined correspondence between a centroid and its corresponding region (triangle) as depicted in Fig. 1] It is important to point out that centroid-based methods have been used in a variety of computer vision applications including clustering. In particular, the K-mean algorithm is one of the most widely centroid-based technique used for clustering [13.

Unless we establish a meaningful measure of distance between the centroids of a triangle mesh, no meaningful exploration of the underlying object structure is possible. In order to take into account the interaction between the centroids, we compute a pairwise distance measure $g\left(\boldsymbol{c}_{i}, \boldsymbol{c}_{j}\right)$ from any centroid $\boldsymbol{c}_{i}$ to all the other centroids $\boldsymbol{c}_{j} \in \mathcal{C}$. Fig. 1 illustrates an arbitrary distance between two centroids. Notice that the distance $g$ need not be an Euclidean metric.

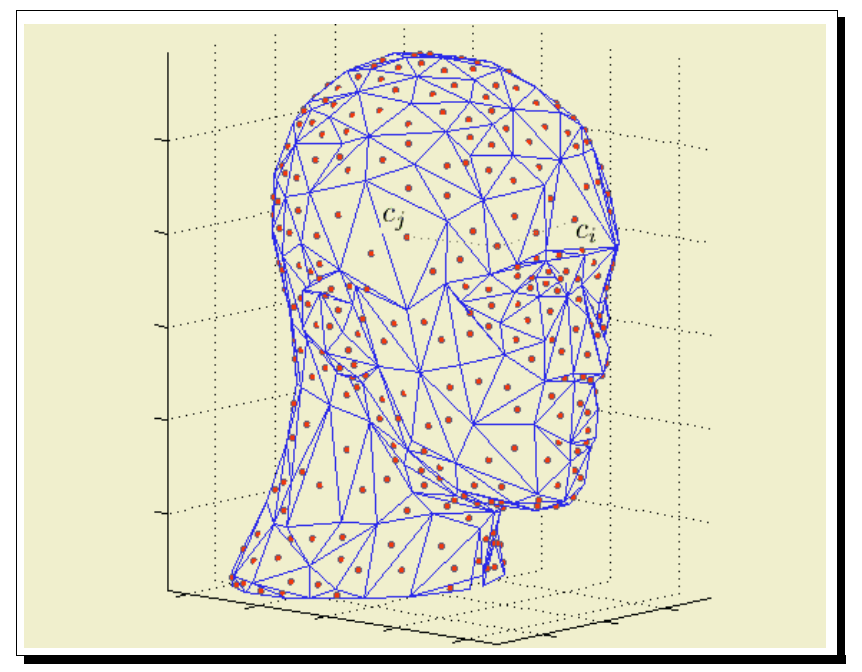

Fig. 1. Distance between two arbitrary centroids of a 3D human head.

To obtain a global measure of the shape $\mathbb{M}$, we simply integrate over all centroids. More precisely, we define a function $f: \mathcal{C} \subset \mathbb{M} \rightarrow \mathbb{R}$ such that

$$
f\left(\boldsymbol{c}_{i}\right)=\frac{1}{|\mathcal{C}|} \int_{\boldsymbol{c}_{j}} g\left(\boldsymbol{c}_{i}, \boldsymbol{c}_{j}\right) d \boldsymbol{c}_{j},
$$

where $d \boldsymbol{c}_{j}$ denotes the area element that contains the centroid $\boldsymbol{c}_{j}$, that is, in the discrete domain, $d \boldsymbol{c}_{j}=\operatorname{area}\left(T_{j}\right)$ the area of the triangle $T_{j}$, and $|\mathcal{C}|=$ 
$\sum_{j=1}^{n}$ area $\left(T_{j}\right)$ is the total area of the surface $\mathbb{M}$. The function $f$ clearly represents a global measure of the shape, and therefore to each triangle mesh $\mathbb{M}$ we will assign its global measure $f$.

The problem addressed in this paper can now be concisely described by the following statement: Given two $3 \mathrm{D}$ objects $\mathbb{M}_{1}$ and $\mathbb{M}_{2}$ to be matched, find their global measures $f_{1}$ and $f_{2}$, and then for a computed dissimilarity measure $D\left(f_{1}, f_{2}\right)$, we are to determine the relative match between them. In other words, the dissimilarity between two objects measures "how different they are", and a smaller value of $D$ means that the two objects are more similar. Fig. 2 depicts a block-diagram of the proposed framework.

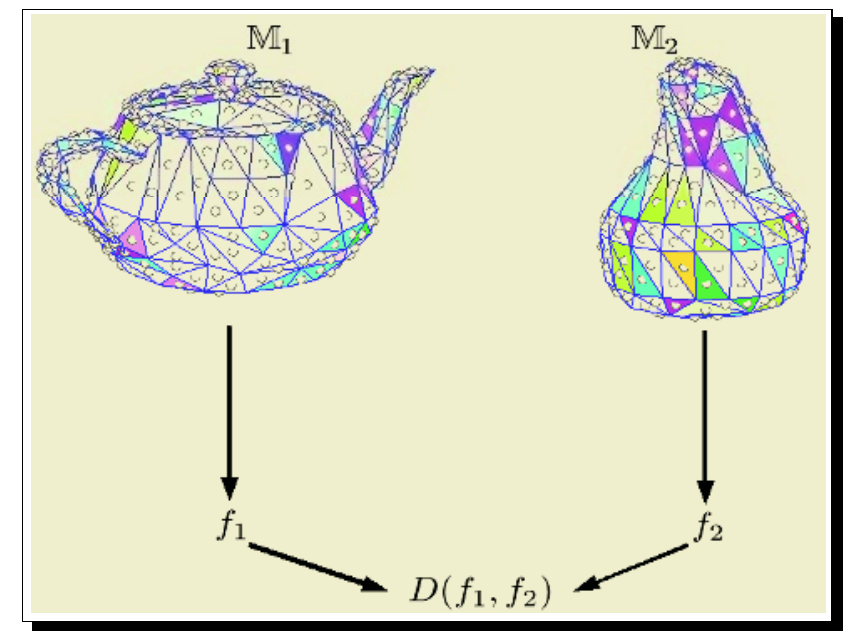

Fig. 2. Block-diagram of the proposed methodology.

\section{Proposed Method}

The basic idea behind the shape descriptor is to characterize a $3 \mathrm{D}$ object with a one-dimensional function which will help us discriminate between objects in a database of 3D models.

\subsection{Global Geodesic Shape Function}

The Reeb graph concept has been shown to be very effective in modeling 3D objects based on cross sections such as MRI or CT images. It is more adapted to modeling applications where the height is of special interest such as terrain imaging. The height function, however, has some limitations to be used as an object signature for matching, indexing, or retrieval of arbitrary 3D objects. 
The main reason is that the height function is not rotationally invariant. To overcome these limitations, we propose a global geodesic function defined on the object surface as follows. Let $\boldsymbol{c}_{i}$ and $\boldsymbol{c}_{j}$ be two points (centroids) on a triangle mesh $\mathbb{M}$. The geodesic distance $g\left(\boldsymbol{c}_{i}, \boldsymbol{c}_{j}\right)$ between $\boldsymbol{c}_{i}$ and $\boldsymbol{c}_{j}$ is the shortest length $L(\gamma)=\int_{a}^{b}\left\|\gamma^{\prime}(t)\right\| d t$ of a smooth curve $\gamma:[a, b] \rightarrow \mathbb{M}$ such that $\gamma(a)=\boldsymbol{c}_{i}$ and $\gamma(b)=\boldsymbol{c}_{j}$. The geodesic distance may be viewed locally as the Euclidean one $d_{E}\left(\boldsymbol{c}_{i}, \boldsymbol{c}_{j}\right)=\left\|\boldsymbol{c}_{i}-\boldsymbol{c}_{j}\right\|$, and it is clearly invariant to rigid transformations. Inspired by the geodesic-based representation for 3D topology matching proposed by Hilaga et al. 12, we define a global shape function $f: \mathcal{C} \rightarrow \mathbb{R}$ expressed in terms of the rotationally invariant (square) geodesic distance as follows

$$
f\left(\boldsymbol{c}_{i}\right)=\frac{1}{|\mathcal{C}|} \int_{\boldsymbol{c}_{j}} g\left(\boldsymbol{c}_{i}, \boldsymbol{c}_{j}\right)^{2} d \boldsymbol{c}_{j} .
$$

The primary motivation behind the geodesic distance is that of overcoming the limitations of the Euclidean distance which is linear in nature and therefore cannot find nonlinear structures in the underlying object. It is worth noting that the geodesic distance in not only rotationally invariant, but also invariant to isometric transformations of the surface [16].

Unlike the Euclidean distance which is basically a straight line between two points in a $3 \mathrm{D}$ space, the geodesic distance captures the global nonlinear structure and the intrinsic geometry of the data as illustrated in Fig. 3. It is clear that the Euclidean distance between two arbitrary points in a nonlinear manifold is just a straight segment connecting both points, whereas the geodesic distance, which is the shortest curve along the surface connecting both points, clearly reflects the nonlinear structure of the object.

Geodesic Distance Calculation. Given the set of centroids $\mathcal{C}=\left\{\boldsymbol{c}_{1}, \ldots, \boldsymbol{c}_{n}\right\}$ of a triangle mesh $\mathbb{M}$, the geodesic distance calculation is based on a graph-based approach recently used to compute the isometric feature mapping (Isomap) for multidimensional scaling on nonlinear manifolds [14]. The algorithm has two main steps:

(i) Construct a neighborhood graph by connecting a given centroid to its $k$-nearest neighbors, and link these neighboring centroids by edges with weights equal to the Euclidean distances.

(ii) Compute the geodesic distances (shortest paths) between all pairs of $n$ points in the constructed graph using Dijkstra's or Floyd's algorithm.

Note that the geodesic distance on triangulated surfaces may also be effectively computed using the fast marching method introduced by Kimmel and Sethian [17.

In discrete form, the geodesic shape function is reduced to

$$
f\left(\boldsymbol{c}_{i}\right)=\frac{1}{|\mathcal{C}|} \boldsymbol{G} \boldsymbol{a}=\boldsymbol{G} \frac{\boldsymbol{a}}{\|\boldsymbol{a}\|_{1}},
$$




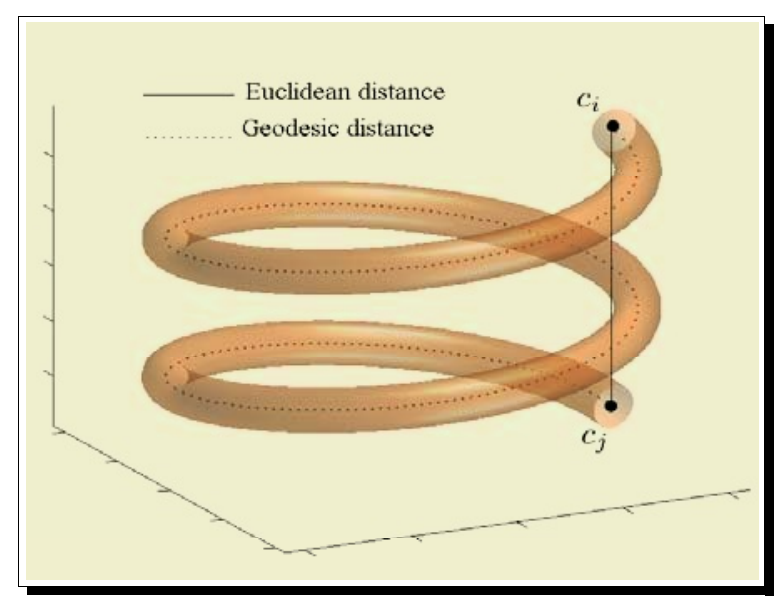

Fig. 3. Euclidean vs. geodesic distance on a nonlinear manifold.

where $\boldsymbol{G}=\left(g_{i j}^{2}\right)$ is the (square) geodesic distance matrix of size $n \times n$, and $\boldsymbol{a}=\left(a_{1}, \ldots, a_{n}\right)^{T}$ is an $n \times 1$ vector of triangle areas, i.e. $a_{j}=\operatorname{area}\left(T_{j}\right)$. The matrix $\boldsymbol{G}=\left(g_{i j}^{2}\right)$ is symmetric with zeros in the diagonal, and positive offdiagonal elements. Note that the geodesic shape function can be expressed as a geodesic shape vector $\mathcal{X}=\left\{X_{1}, \ldots, X_{n}\right\}$, where $X_{i}=f\left(\boldsymbol{c}_{i}\right)$. This vector may be viewed as a shape descriptor that can be used for $3 \mathrm{D}$ shape comparison.

\subsection{Global Geodesic Shape Distribution}

Assume that the geodesic shape vector $\mathcal{X}$ of an object $\mathbb{M}$ is a random sample with a common (unknown) probability density function $p$. A common approach to approximating the probability density function $p$ is through the kernel density estimation. This is an important data-driven tool that provides a very effective way of unraveling structure in a set of data [15]. The kernel density estimator $\hat{p}$ is given by

$$
\hat{p}(x)=\frac{1}{n h} \sum_{i=1}^{n} \mathcal{K}\left(\frac{x-X_{i}}{h}\right),
$$

where $\mathcal{K}$ is a Gaussian kernel, and $h$ is the bandwidth or window width to be estimated. A good selection of this bandwidth is given by (see [15])

$$
\hat{h}=\left[\frac{243 R(\mathcal{K})}{35 \mu_{2}(\mathcal{K})^{2} n}\right]^{1 / 5} \hat{\sigma},
$$

where $R(\mathcal{K})=\int \mathcal{K}(t)^{2} d t, \mu_{2}(\mathcal{K})=\int t^{2} \mathcal{K}(t) d t$, and $\hat{\sigma}=\operatorname{med}_{j}\left\{\left|X_{j}-\operatorname{med}_{i}\left\{X_{i}\right\}\right|\right\}$ is the median absolute deviation.

To each $3 \mathrm{D}$ object represented by a triangle mesh $\mathbb{M}$, we therefore associate a kernel density $\hat{p}$ which we will refer to as geodesic shape distribution. This 
probabilistic shape descriptor represents the object information and will be used in our matching experiments. In order to compare two geodesic shape distributions and hence to measure the performance of the proposed scheme, we will describe in the next section an information-theoretic distance that quantifies the difference between two 3D shapes through their probabilistic shape descriptors.

\section{Probabilistic Dissimilarity}

Let $\mathbb{M}_{1}$ and $\mathbb{M}_{2}$ two $3 \mathrm{D}$ objects with geodesic shape distributions $\hat{p}$ and $\hat{q}$ respectively. Information theoretic measures provide quantitative entropic divergences between two probability distributions. A common entopic measure is KullbackLeibler divergence. This dissimilarity measure, however, is not symmetric, unbounded, and undefined if $\hat{p}$ is not absolutely continuous with respect to $\hat{q}$. To overcome these limitations, we use the Jensen-Shannon divergence $D$ defined as

$$
D(\hat{p}, \hat{q})=H\left(\frac{\hat{p}+\hat{q}}{2}\right)-\frac{H(\hat{p})+H(\hat{q})}{2},
$$

where $H(\hat{p})=-\int \hat{p}(x) \log _{2} \hat{p}(x) d x$ is the differential entropy.

The Jensen-Shannon divergence is a statistical distance that is very useful in quantifying differences between probability distributions or densities. In other words, this dissimilarity measure quantifies differences in shape between two arbitrary objects.

Unlike the Kullback-Leibler divergence, the Jensen-Shannon divergence has the advantage of being symmetric, always defined, and generalizable to any number of probability distributions, with a possibility of assigning weights to these distributions 9]. In addition to its convexity property, the Jensen-Shannon divergence is shown to be an adapted measure of disparity among probability distributions, and it is upper bounded $D(\hat{p}, \hat{q}) \leq \log _{2}(2)=1$ (see [11] for more details).

\section{Experimental Results}

Object matching experiments were performed using a database of 3D models collected from the internet. Each model is represented as a triangle mesh, and the number of centroids is chosen to be equal $n=1000$ for all models used in experimentation. This number was fixed by reducing the number of triangles while trying to preserve the overall shape of the triangle mesh. We experimented with different values of $n$, and the resulting geodesic shape distribution has been shown to be relatively insensitive to the choice of the number of centroids.

We conducted two sets of experiments. The first set of simulations deals with objects that are topologically equivalent to a sphere (i.e. with genus equal to zero) as shown in Fig. 4 The numerical results using the Jensen-Shannon dissimilarity measure are depicted in Table 1 where the grayscale colorbar displays the grayscale colormap of this dissimilarity matrix. This grayscale colormap ranges from white (maximum similarity) to black (maximum dissimilarity), and passes 

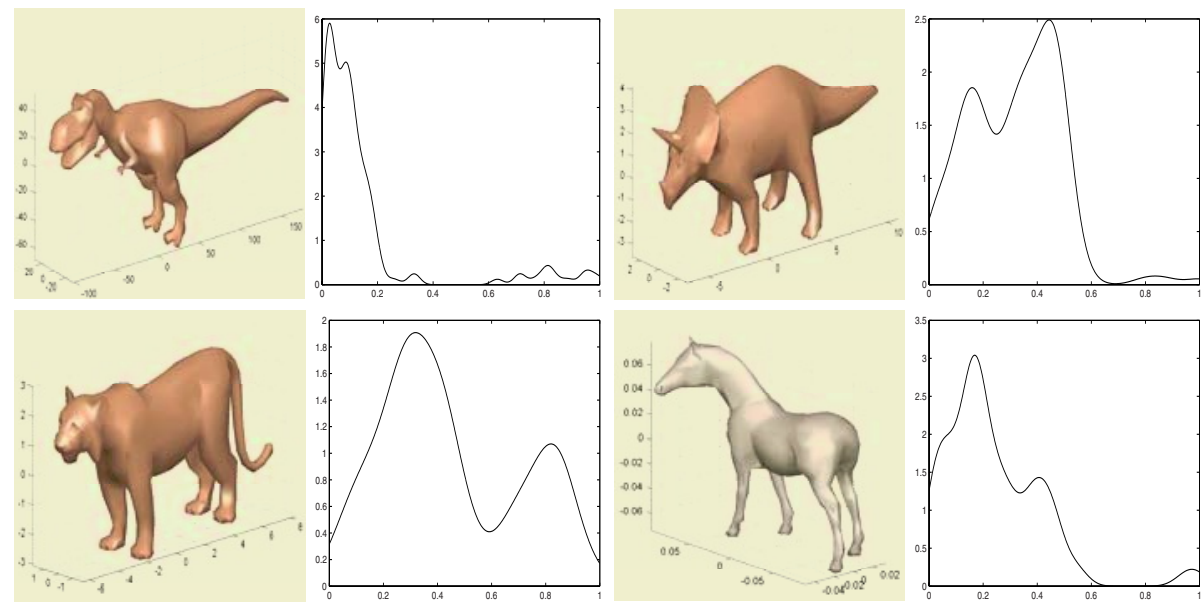

Fig. 4. First set of experiments: 3D models and their geodesic shape distributions.

Table 1. Jensen-Shannon dissimilarity results for the first set of experiments.

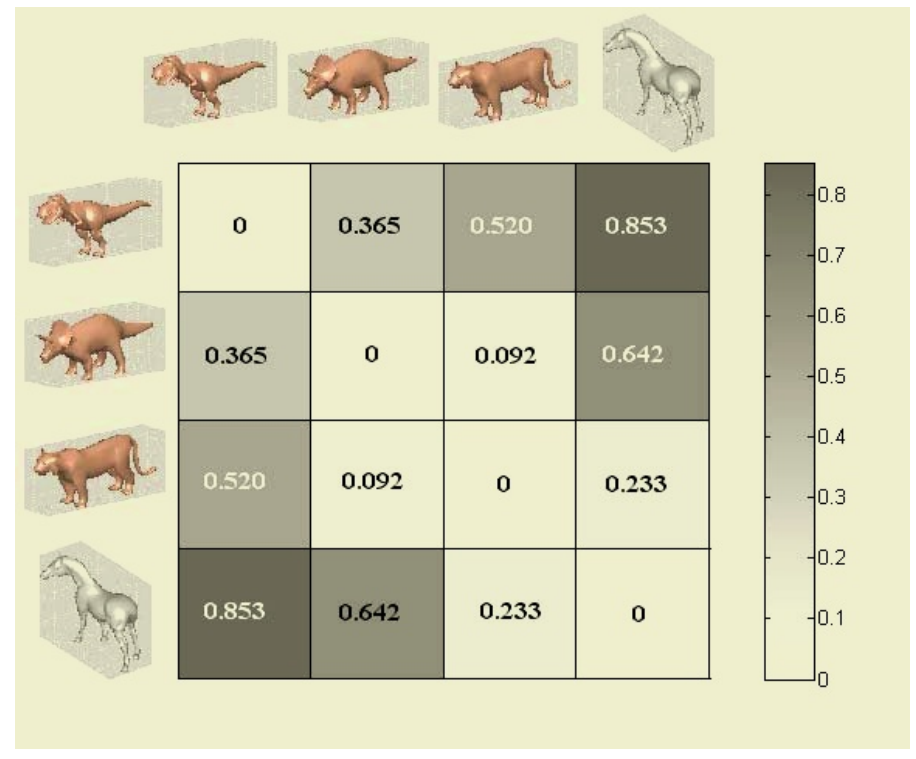

through the gray colors indicating the values of the matching performance. As illustrated in Table 1, the minimum dissimilarity rate is about $9 \%$, that is the matching rate is about $91 \%$.

The second set of experiments is similar the first, except that the underlying objects have a different topology than the ones in the first part of the experiments. Fig. 5 shows a set of objects with genus equal to one. Matching is achieved by the minimum Jensen-Shannon distance computations as illustrated 

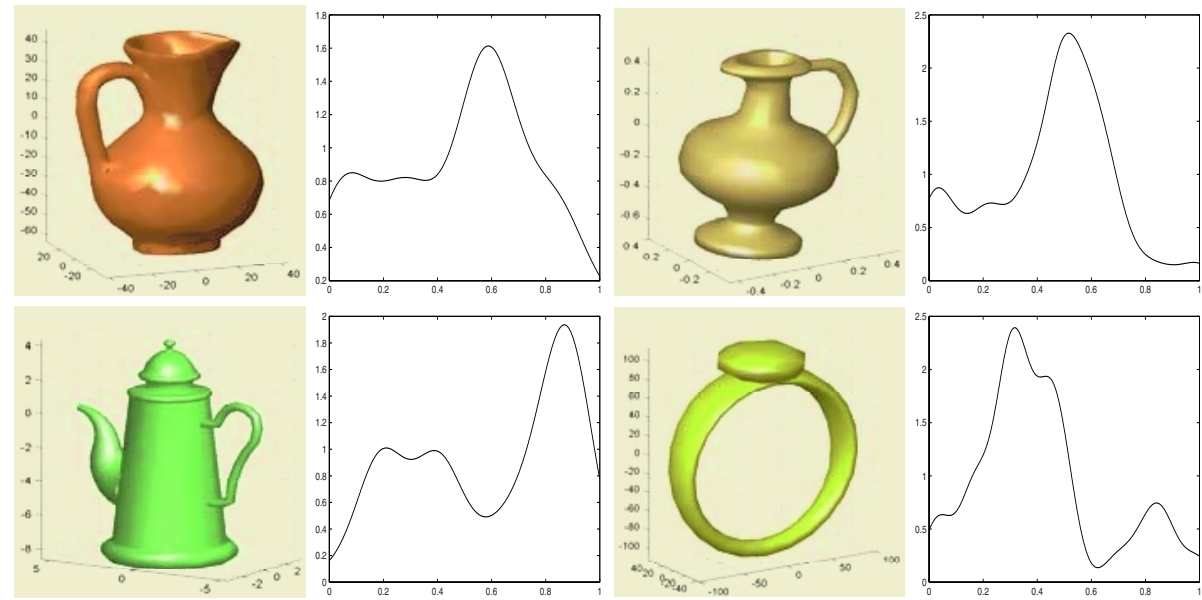

Fig. 5. Second set of experiments: 3D models and their geodesic shape distributions.

Table 2. Jensen-Shannon dissimilarity results for the second set of experiments.

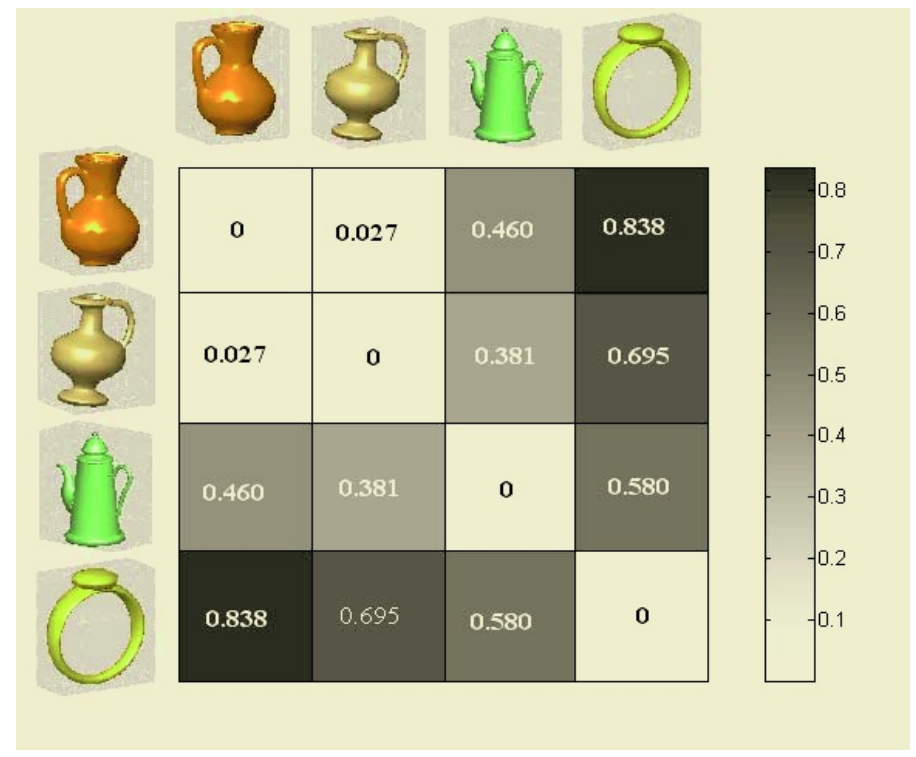

in Table 2. Note that the minimum dissimilarity rate is about $2 \%$, that is the matching rate is about $98 \%$.

\section{Conclusions and Future Work}

In this paper, we proposed an new methodology for object matching. The key idea is to encode a $3 \mathrm{D}$ shape into a $1 \mathrm{D}$ geodesic shape distribution. Object match- 
ing is then achieved by calculating an information-theoretic measure of dissimilarity between the probability distributions. That is, the dissimilarity computations are carried out in a low-dimensional space of geodesic shape distributions. Finally, while the experimental results presented in this paper are very encouraging, significant additional performance gains and efficient parallelizations are still possible.

\section{References}

1. Y. Shinagawa, T.L. Kunii, and Y.L. Kergosien, "Surface coding based on Morse theory," IEEE Comp. Graph. and Appl., vol. 11 no. 5 , pp. 66-78, 1991.

2. A.T. Fomenco and T.L. Kunii, Topological modeling for visualization, SpringerVerlag Tokyo, 1997.

3. J.J. Koenderink, Solid Shape, MIT Press, Cambridge, Massachusetts, 1990.

4. J.W. Bruce and P.J. Giblin, Curves and singularities, Cambridge University Press, second edition, 1992.

5. C. Lu, Y. Cao, and D. Mumford, "Surface evolution under curvature flows," J. of Vis. Commun. and Image Representation, vol. 13, no. 1/2, pp. 65-81, 2002.

6. R. Osada, T. Funkhouser, B. Chazelle, and D. Dobkin, "Shape distributions," ACM Transactions on Graphics, vol. 21, no. 4, pp. 807-832, October 2002.

7. S. Kullback and R. Liebler, "On information and sufficiency," Ann. Math. Statist., vol. 22, pp. 79-86, 1951.

8. R. Stoica, J. Zerubia, and J.M. Francos, "Image retrieval and indexing: A hierarchical approach in computing the distance between textured images," IEEE Int. Conf. on Image Processing, Chicago, 1998.

9. J. Lin, "Divergence measures based on the Shannon entropy," IEEE Trans. Information Theory, vol. 37, no. 1, pp. 145-151, 1991.

10. A.O. Hero, B. Ma, O. Michel and J. Gorman, "Applications of entropic spanning graphs," IEEE Signal Processing Magazine, vol. 19, pp. 85-95, Sept. 2002.

11. Y. He, A. Ben Hamza, and H. Krim, "A generalized divergence measure for robust image registration", IEEE Trans. on Signal Processing, vol. 51, no. 5, May 2003.

12. M. Hilaga, Y. Shinagawa, T. Komura, and T.L. Kunii, "Topology matching for fully automatic similarity estimation of 3D shapes,", Proc. SIGGRAPH, pp. 203-212, August 2001.

13. J. MacQueen, "Some methods for classification and analysis of multivariate observations," Proc. 5th Berkeley Symp. Math. Stat. and Prob., 1967.

14. J.B. Tenenbaum, V. de Silva, and J.C. Langford, "A global geometric framework for nonlinear dimensionality reduction," Science, vol. 290, pp. 2319-2323, Dec. 2000.

15. M.P. Wand and M.C. Jones, Kernel smoothing, Chapman and Hall, London, 1995.

16. A. Elad and R. Kimmel, "Bending invariant representations for surfaces," Proc. of Computer Vision and Pattern Recognition, pp. 168-174, 2001.

17. R. Kimmel and J.A. Sethian, "Computing geodesic on manifolds," Proc. of National Academy of Science, 95, pp. 8431-8435, 1998. 